\title{
Habilidades de resolução de conflito e ocorrência de disfluências comuns em crianças em desenvolvimento normal de linguagem
}

\author{
Conflict resolution abilities and normal disfluencies in normally \\ developing children
}

\author{
Debora Maria Befi-Lopes ${ }^{1}$, Erica Macêdo de Paula²
}

\begin{abstract}
RESUMO
Objetivos: (1) Investigar as habilidades de resolução de conflito (RC) de crianças em idade escolar; (2) Verificar a relação entre a ocorrência de disfluências comuns (DC) e o desenvolvimento das habilidades de RC. Métodos: Participaram do estudo 20 crianças em desenvolvimento normal de linguagem, com idades entre sete e dez anos. As crianças foram submetidas a uma triagem, na qual foram realizadas as provas de Fonologia, Consciência Fonológica e Leitura e Escrita; aquelas que obtiveram desempenho esperado para sua faixa etária passaram por avaliação, sendo realizadas as provas de RC e de Fluência. Na prova de RC, as respostas foram categorizadas em 28 categorias, para então, serem pontuadas. Na prova de fluência, foi verificada a ocorrência de DC. Resultados: Na prova de RC, as crianças obtiveram entre 5 e 14 pontos $(\mathrm{M}=10,7)$, porém não se observou correlação entre a pontuação total e a idade cronológica ( $\mathrm{p}=0,361)$. Na prova de Fluência, a ocorrência de DC variou de 4 a $24(\mathrm{M}=10)$. Observou-se que não há correlação estatística significante entre a pontuação na prova de RC e a ocorrência de DC ( $\mathrm{p}=0,899)$. Conclusões: Na prova de RC, as crianças utilizaram principalmente estratégias unilaterais para a resolução dos conflitos e as estratégias não se tornaram mais sofisticadas com o passar da idade. Ao comparar o desempenho das crianças na prova de RC à ocorrência de DC, não foi possível estabelecer relação direta entre as duas variáveis.
\end{abstract}

Descritores: Cognição; Linguagem infantil; Desenvolvimento da linguagem; Comunicação; Fala

\section{INTRODUÇÃOO}

\section{Resolução de conflito}

Cognição social é um importante aspecto da cognição, que consiste na compreensão do mundo social, incluindo a compreensão sobre o conhecimento, pensamento, intenções, emoções e pontos de vista do outro, além do conhecimento sobre papéis e relações sociais ${ }^{(1)}$.

Atualmente, ainda existem dúvidas sobre a exata relação entre o desenvolvimento da linguagem e da cognição social.

Trabalho realizado no Laboratório de Investigação Fonoaudiológica em Desenvolvimento da Linguagem e suas Alterações do Curso de Fonoaudiologia da Faculdade de Medicina da Universidade de São Paulo - USP - São Paulo (SP), Brasil.

(1) Livre Docente, Professora do Departamento de Fisioterapia, Fonoaudiologia e Terapia Ocupacional da Faculdade de Medicina da Universidade de São Paulo - USP - São Paulo (SP), Brasil.

(2) Pós-graduanda do Programa de Pós-graduação em Ciências da Reabilitação da Faculdade de Medicina da Universidade de São Paulo - USP - São Paulo (SP), Brasil.

Endereço para correspondência: Debora Maria Befi-Lopes. R. Cipotânea, 51, Cidade Universitária, São Paulo - SP, CEP: 05360-160. E-mail: dmblopes@usp.br

Recebido em: 27/2/2007; Aceito em: 27/5/2008
Alguns autores ${ }^{(2)}$ acreditam que habilidades sócio-cognitivas são a base para a aquisição da linguagem; outros $^{(3)}$ sugerem que tanto o desenvolvimento da linguagem, quanto da cognição social são decorrentes de habilidades cognitivas mais básicas e da habilidade para discriminar, codificar e arquivar informações necessárias para o desenvolvimento de representações lingüísticas e sócio-cognitivas.

O desenvolvimento da cognição social é necessário para que possamos interagir adequadamente com outros indivídu$\mathrm{os}^{(4-5)}$. Um tipo de interação social, que caracteriza o desenvolvimento cognitivo, é a resolução de conflito. Para resolver conflitos é preciso compreender o ponto de vista do outro, assim como desenvolver e expressar estratégias para resolver disputas. Crianças pequenas ou com déficit na cognição social utilizam estratégias de resolução de conflito mais globais e menos sofisticadas, pois muitas vezes, não são capazes de compreender o ponto de vista do seu oponente ${ }^{(1,6)}$.

Diversos estudos relatam déficit de cognição social em crianças com Distúrbio Específico de Linguagem (DEL), sugerindo, portanto, uma relação entre linguagem e cognição social $^{(7-10)}$. Crianças com DEL não são capazes de utilizar determinadas regras lingüísticas para necessidades comunicativas específicas como, por exemplo, a resolução de conflitos ${ }^{(11)}$.

Em estudo realizado na década de 90, esta habilidade es- 
pecífica foi estudada. Os autores verificaram como crianças com DEL, com idades entre oito anos e cinco meses e 12 anos e dez meses, e crianças em desenvolvimento normal de linguagem, com idades entre oito anos e quatro meses e 12 anos e dez meses, resolvem conflitos em situações hipotéticas e em situações reais (encenação). Crianças com DEL usaram um menor número de estratégias e estratégias mais primitivas para resolver conflitos nas situações hipotéticas, mas não nas encenações $^{(1)}$.

Nesta mesma pesquisa, os autores verificaram que, no grupo composto por crianças em desenvolvimento normal de linguagem, não houve uma diferença estatisticamente significativa, entre os sexos e entre as idades, no que se refere à qualidade das respostas ${ }^{(1)}$.

\section{Fluência}

Em artigo publicado anteriormente, a autora relata que: "a fala fluente ocorre quando há um alto grau de continuidade e a disfluência; por sua vez, são as rupturas no fluxo da fala, que variam dependendo do dia, da emoção envolvida e do domínio sobre o tema da conversação" ${ }^{(12)}$.

As disfluências são observadas na fala de todos os indivíduos; não existe uma fala $100 \%$ fluente, porém a literatura refere que as disfluências encontradas na fala dos indivíduos fluentes diferem, qualitativamente, das disfluências observadas na fala dos sujeitos gagos ${ }^{(12-13)}$. As tipologias das rupturas podem ser classificadas da seguinte forma ${ }^{(12,14)}$ :

Tipo comum: hesitação, interjeição, revisão, palavras não terminadas, repetição de segmento e repetição de frase.

Tipo não-comum: duas ou mais repetições de sons e/ ou sílabas e/ou palavras, prolongamento, bloqueio, pausa e intrusão.

A ocorrência de disfluências comuns está relacionada, entre outros fatores, à demanda lingüística; portanto, em situações descontextualizadas, como a resolução de conflito, é provável que ocorram mais disfluências do que em situações contextualizadas, como o reconto de uma história ${ }^{(15)}$.

O desenvolvimento da fala fluente está diretamente relacionado a outras áreas da comunicação, como o desenvolvimento da competência lingüística, que envolve a aquisição e o desenvolvimento adequado do vocabulário, da sintaxe, da morfologia e da fonologia; e a habilidade de utilizar esses aspectos da linguagem em situações de comunicação ${ }^{(16-17)}$.

Devido à relação entre fluência e linguagem, observa-se, muitas vezes, na fala de crianças com DEL uma grande ocorrência de disfluências comuns. As disfluências observadas no DEL podem estar relacionadas à dificuldade dessa população em elaborar uma mensagem e organizá-la em estruturas frasais adequadas ou à diferença entre a demanda lingüística e a capacidade de elaboração e produção ${ }^{(18)}$.

Outro estudo buscou verificar a ocorrência de disfluências no discurso de 22 pares de crianças de nove anos de idade, com e sem DEL. As crianças com histórico de dificuldades lingüísticas, apresentaram significativamente mais disfluências em seus discursos ${ }^{(19)}$.

Observa-se, também, uma importante relação entre fluência e discurso. Indivíduos que apresentam dificuldades de acesso lexical ou dificuldades de elaboração discursiva podem apresentar em sua fala um grande número de disfluências comuns, comprometendo a coerência discursiva e a transmissão da mensagem $^{(18,20)}$.

O desenvolvimento da habilidade discursiva é um dos grandes marcos, na aquisição de linguagem; este processo inicia-se cedo, apenas alguns meses após a criança produzir suas primeiras frases. Por volta dos 22 meses, com assistência dos adultos, as crianças começam a se referir a eventos passados, e entre três e cinco anos, começam a contar narrativas mais longas e complexas ${ }^{(21)}$.

\section{Resolução de conflito e fluência}

As habilidades de resolução de conflito são importantes parâmetros para averiguar a cognição social de um indivíduo e algumas importantes habilidades lingüísticas, como a capacidade de elaborar e expressar uma resolução adequada para determinada situação. Assim como a capacidade de resolver conflitos, a habilidade de elaborar um discurso fluente também fornece importantes dados sobre a competência lingüística de um indivíduo. A fim de investigar a relação entre um discurso fluente (ocorrência de disfluências comuns) e as habilidades de resolução de conflito este estudo será realizado.

Os objetivos do presente estudo são:

1- Verificar as habilidades de resolução de conflito de crianças em idade escolar.

2 - Verificar a relação entre a ocorrência de disfluências comuns e as habilidades de resolução de conflito.

\section{MÉTODOS}

Participaram do estudo 20 crianças em desenvolvimento normal de linguagem, com idades entre sete e dez anos (cinco crianças por faixa etária), selecionadas de uma escola estadual de ensino fundamental.

No Quadro 1 tem-se a descrição geral das crianças que participaram do estudo, caracterizando-as quanto a sexo e idade. Dos 20 participantes, sete (35\%) eram do sexo masculino e 13 $(65 \%)$ do sexo feminino. As idades variaram de $7 \mathrm{a}$ a $10 \mathrm{a} 6 \mathrm{~m}$, sendo a média de idade de $8 \mathrm{a} 9 \mathrm{~m}$.

O material utilizado foi composto por um gravador digital Panasonic RR-US380 e pelas provas de Fonologia, Consciência Fonológica, Leitura e Escrita, Resolução de Conflitos e Fluência ${ }^{(1,14,22-25)}$.

As crianças foram submetidas a uma triagem, onde foram realizadas as provas de Fonologia, Consciência Fonológica e Leitura e Escrita ${ }^{(22-25)}$. Para serem incluídas na pesquisa, as crianças deveriam obter desempenho esperado para sua faixa etária nas três provas da triagem. Seriam excluídas do estudo as crianças que não compreendessem as situações hipotéticas de conflito; porém, todos os sujeitos demonstraram que compreenderam adequadamente as cinco histórias. As crianças selecionadas passaram por avaliação, na qual foram realizadas as provas de Resolução de Conflito e de Fluência. Os testes foram aplicados na escola das crianças, em aproximadamente 30 minutos $^{(1,14)}$.

A prova de Resolução de Conflitos é composta por cinco 
Quadro 1. Caracterização dos sujeitos

\begin{tabular}{|l|c|c|}
\hline Sujeitos & Idade & Sexo \\
\hline S1 & $7 a$ & $F$ \\
\hline S2 & $7 a 2 m$ & M \\
\hline S3 & $7 a 8 m$ & $F$ \\
\hline S4 & $7 a 10 m$ & M \\
\hline S5 & $7 a 11 m$ & M \\
\hline S6 & $8 a 4 m$ & $F$ \\
\hline S7 & $8 a 5 m$ & $F$ \\
\hline S8 & $8 a 8 m$ & M \\
\hline S9 & $8 a 8 m$ & $F$ \\
\hline S10 & $8 a 11 m$ & $F$ \\
\hline S11 & $9 a 2 m$ & $F$ \\
\hline S12 & $9 a 4 m$ & $M$ \\
\hline S13 & $9 a 7 m$ & $F$ \\
\hline S14 & $9 a 7 m$ & $M$ \\
\hline S15 & $9 a 10 m$ & $F$ \\
\hline S16 & $10 a$ & $F$ \\
\hline S17 & $10 a 1 m$ & $F$ \\
\hline S18 & $10 a 2 m$ & M \\
\hline S19 & $10 a 3 m$ & $F$ \\
\hline S20 & $10 a 6 m$ & $F$ \\
\hline
\end{tabular}

histórias (descritas em artigo publicado anteriormente ${ }^{(1)}$ ), todas compostas por apenas três frases (Anexo 1), e por desenhos que representam as situações de conflito; para cada história foram confeccionadas três figuras, representando cada uma das frases. Os desenhos foram julgados por quatro fonoaudiólogas pósgraduadas, que verificaram se as figuras realmente remetiam à história contada. Após o primeiro julgamento, os desenhos referentes a cada uma das histórias foram refeitos a partir das sugestões, foram apresentados novamente às julgadoras e, então, aprovados.

A prova de Resolução de Conflito foi aplicada da seguinte maneira: inicialmente as crianças receberam a seguinte instrução: "Eu gostaria de saber o que meninos (as) como você pensam sobre as coisas. Eu vou contar algumas coisas que aconteceram com um menino. Depois eu gostaria que você pensasse todas as coisas que ele poderia fazer. Me fale tudo o que vier na sua cabeça. Finja que todos os meninos têm [idade do sujeito] anos".

Em seguida, foram apresentados oralmente às crianças os cinco contextos hipotéticos de conflito; ao mesmo tempo, para facilitar a compreensão das crianças, foram expostos os desenhos que representavam as situações de conflito.

Após cada apresentação, as crianças foram instruídas a recontar a história, para verificar a compreensão dos acontecimentos. Logo após, elas deveriam relatar tudo o que o protagonista poderia fazer naquela situação. As respostas foram gravadas em um gravador digital Panasonic RR-US380.

As respostas foram analisadas, para determinar inicialmente a presença ou ausência de estratégias de resolução de conflito. Posteriormente, as respostas foram categorizadas em
28 categorias - baseadas em artigo publicado anteriormente ${ }^{(1)}$ - descritas no Quadro 2.

As categorias, excluindo "outras", foram agrupadas em quatro níveis, formando uma escala evolutiva de desenvolvimento para habilidades de resolução de conflitos.

Nivel 1: Soluções físicas para resolução de conflitos (intervenção física, intervenção verbal e não-interação).

Nivel 2: Soluções unilaterais para resolução de conflitos (suborno material; ameaçar, ordenar ou exigir; castigo como punição; apelar para autoridade do adulto; justificar; reclamar; sarcasmo; exclusão do outro; questionar; iniciativas "fracas"; obedecer a ordens, ceder e pedir desculpas ou apaziguar).

Nivel 3: Soluções cooperativas para resolução de conflitos (pedidos, sugestões; persuadir e explicar; esperar, adiar a ação; punição para ensinar uma lição; suborno interpessoal; conciliação; mudança de perspectiva e empatia, inferência de sentimento, complacência).

Nível 4: Soluções mútuas para resolução de conflitos (discussão, apelar para unidade e soluções conjuntas).

Após terem sido agrupadas, as respostas foram pontuadas da seguinte maneira:

$\begin{array}{ll}\text { s } & \text { Outras }-0 \text { ponto } \\ \mathrm{s} & \text { Nivel } 1-1 \text { ponto } \\ \mathrm{s} & \text { Nivel } 2 \text { - } 2 \text { pontos } \\ \mathrm{s} & \text { Nivel } 3-3 \text { pontos } \\ \mathrm{s} & \text { Nível } 4-4 \text { pontos }\end{array}$

Para verificar a fluência do discurso, os sujeitos realizaram a prova de Fluência do Teste de Linguagem Infantil ABFW ${ }^{(14)}$. As crianças foram expostas a uma gravura e instruídas a contar uma história. O discurso deveria ter, no mínimo, 200 sílabas expressas (sílabas não disfluentes) para ser analisado; para os discursos com menos de 200 sílabas fluentes, foi aplicada uma regra de três para compatibilização ${ }^{(14)}$. Os discursos foram gravados em um gravador digital Panasonic ${ }^{\circledR}$ RR-US380, para serem analisados posteriormente.

No discurso realizado pelas crianças, foi verificado o número de disfluências comuns, pois esse parâmetro fornece importantes dados sobre a efetividade do discurso e a competência lingüística dos sujeitos.

Para a análise estatística deste trabalho, foram utilizados os testes paramétricos de ANOVA, Correlação de Pearson e Teste de Correlação. Na complementação da analise descritiva, fizemos uso da técnica de Intervalo de Confiança para média. Para este trabalho, foi adotado um nível de significância de $0,05(5 \%)$, sendo todos os intervalos de confiança construídos com $95 \%$ de confiança estatística.

A presente pesquisa foi aprovada pela Comissão de Pesquisa do Departamento de Fisioterapia, Fonoaudiologia e Terapia Ocupacional da Faculdade de Medicina da Universidade de São Paulo sob o número 059/06, e os responsáveis pelas crianças participantes assinaram o Termo de Consentimento Livre e Esclarecido.

\section{RESULTADOS}

A seguir, serão descritos os resultados obtidos pelos 20 participantes da pesquisa, nas provas de Resolução de Conflito e Fluência. 
Quadro 2. Categorias de estratégias de resolução de conflito para situações hipotéticas

\begin{tabular}{|c|c|c|}
\hline Níveis Evolutivos & Estratégia & Definição \\
\hline \multirow{3}{*}{ Nível 1} & Intervenção física & Uso da força, ex. empurrar, bater \\
\hline & Intervenção verbal & Xingamentos e insultos \\
\hline & "Não-interação" & Se retirar ou evitar o outro \\
\hline \multirow{12}{*}{ Nível 2} & Suborno material & Subornos por objetos \\
\hline & Ameaçar, ordenar ou reivindicar & Exigir a ação de alguém \\
\hline & Castigo como punição & Punir o outro indivíduo \\
\hline & Apelar para autoridade do adulto & Procurar a ajuda de um adulto para resolução do conflito \\
\hline & Justificar & $\begin{array}{l}\text { Justificar, dizendo que estava no lugar primeiro ou que tinha a } \\
\text { permissão de um adulto }\end{array}$ \\
\hline & Reclamar & Expressão de insatisfação ou ressentimento \\
\hline & Sarcasmo & Expressões sarcásticas \\
\hline & Exclusão do outro & Não refletir sobre o outro indivíduo \\
\hline & Questionar & Questionar para resolver os conflitos \\
\hline & Iniciativas "fracas" & Uso de "por favor", se fazer de vítima ou de fraco \\
\hline & Obedecer à ordens, ceder & Uso de poder \\
\hline & Pedir desculpas, apaziguar & Tentativas de amenizar a situação para o outro indivíduo \\
\hline \multirow{8}{*}{ Nível 3} & Pedidos, sugestões, instruções & Perguntar, dar dicas, instruções \\
\hline & Persuadir e explicar & Apelar para a capacidade racional do outro \\
\hline & Esperar, adiar a ação & Adiar a resolução \\
\hline & Punição para ensinar uma lição & Providenciar um castigo, para favorecer um possível aprendizado \\
\hline & Suborno interpessoal & Suborno psicológico, não material \\
\hline & Conciliação & Troca de turno, mediar e dividir \\
\hline & Mudança de perspectiva & Levar em conta o ponto de vista do outro \\
\hline & $\begin{array}{l}\text { Empatia, inferência de sentimento / } \\
\text { complacência }\end{array}$ & Inferir sobre o sentimento do outro \\
\hline \multirow{4}{*}{ Nível 4} & Discussão & Conversar sobre o conflito \\
\hline & Apelar para unidade & Apelar para um grupo ou "unidade" \\
\hline & Soluções conjuntas & $\begin{array}{l}\text { Soluções que envolvem decisão mútua ou negociações } \\
\text { interpessoais }\end{array}$ \\
\hline & Outras & $\begin{array}{l}\text { Todas as estratégias que não encaixam em nenhuma das demais } \\
\text { categorias }\end{array}$ \\
\hline
\end{tabular}

Na prova de Resolução de Conflito, três crianças (S3, S13 e S20) obtiveram 14 pontos, a máxima pontuação neste estudo. Uma criança (S15) obteve 13 pontos, três crianças (S6, S14 e S17) obtiveram 12 pontos, três (S4, S10 e S16) obtiveram 11 pontos; quatro ( $\mathrm{S} 1, \mathrm{~S} 7, \mathrm{~S} 18$ e $\mathrm{S} 19)$ obtiveram 10 pontos, cinco (S2, S5, S8, S9 e S11) obtiveram 9 pontos e somente uma criança (S12) obteve apenas 5 pontos.

A análise estatística mostrou que não há correlação entre a pontuação total na prova de Resolução de Conflito e a idade cronológica $(\mathrm{p}=0,361)$. Este dado pode ser observado na Figura 1.

Nesta pesquisa, cada criança participante sugeriu uma solução para cada situação hipotética; a Tabela 1 mostra o número de estratégias sugeridas que pertenciam aos níveis um, dois, três, quatro e à categoria "outras". Nota-se que a maioria das estratégias (41\%) pertencia ao Nível 2; 29\% das

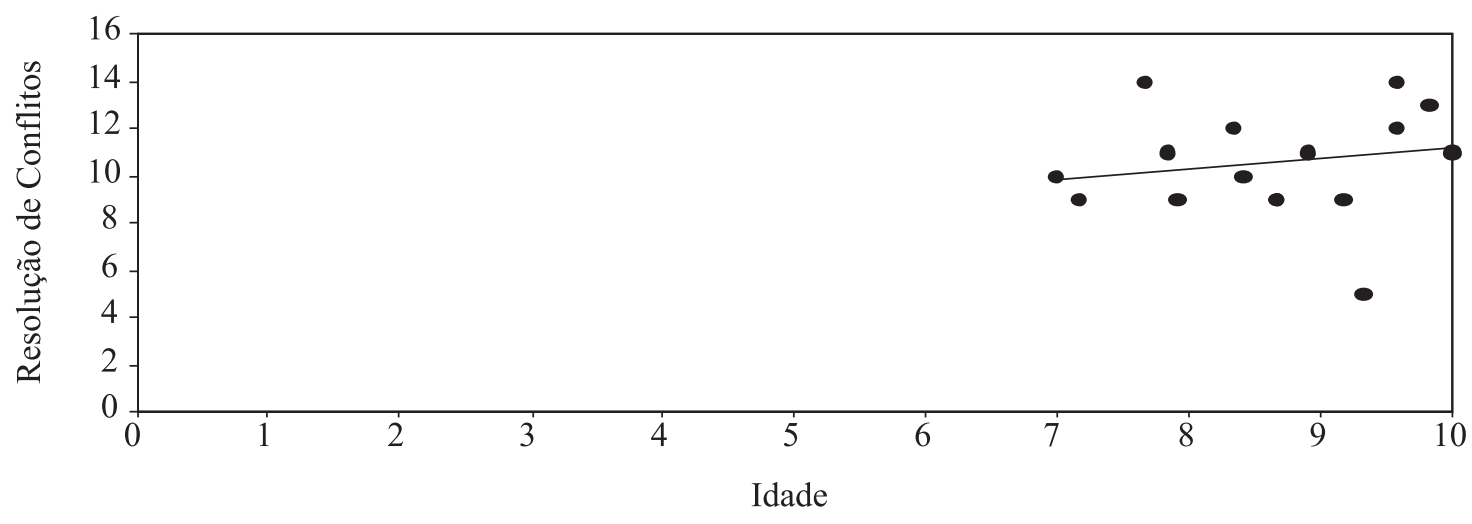

Figura 1. Total na prova de Resolução de Conflito X idade 
Tabela 1. Estratégias de resolução de conflito por nível de desenvolvimento

\begin{tabular}{lc}
\hline Níveis de Resolução de Conflito & Número de soluções sugeridas \\
\hline Nível 1 & 21 \\
Nível 2 & 41 \\
Nível 3 & 29 \\
Nível 4 & 6 \\
"Outras" & 3 \\
\hline
\end{tabular}

estratégias sugeridas pertenciam ao Nível 3, $21 \%$ ao Nível 1, $6 \%$ ao Nível 4 e apenas $3 \%$ à categoria "outras".

Com relação ao desempenho das meninas e dos meninos na tarefa de Resolução de Conflito, a análise estatística mostra que houve diferença estatística significante $(\mathrm{p}=0,031)$ entre a pontuação média das meninas $(\mathrm{M}=11,46)$ e dos meninos $(\mathrm{M}=9,29)$, sendo que as meninas possuem uma média maior do que os meninos.

$\mathrm{N}$ a prova de Fluência, uma criança, S2, apresentou grande ocorrência de disfluências comuns, com um total de 24 disfluências. No discurso do sujeito S17 ocorreram 17,5 disfluências comuns. Os sujeitos S3, S4 e S7 apresentaram em seus discursos 13 disfluências comuns; logo em seguida aparecem os sujeitos S6, S9, S14 e S16 que apresentaram entre, 10 e 12 disfluências comuns. No discurso dos sujeitos S5, S8, S11, S18 e S20, ocorreram entre 8 e 9,4 disfluências comuns. Os sujeitos S13 e S19 realizaram discursos com 7 disfluências comuns. Os sujeitos S10 e S1, realizaram discursos com 5 e 6 disfluências comuns respectivamente. Nos discursos dos sujeitos S12, S15, ocorreram apenas 4 disfluências comuns.

Ao comparar o desempenho das crianças nas provas de Resolução de Conflito e Fluência, a análise estatística evidenciou que não há correlação estatística significante entre a pontuação na prova de Resolução de Conflito e a ocorrência de disfluências comuns $(\mathrm{p}=0,899)$. Este dado pode ser observado na Figura 2.

\section{DISCUSSÃO}

Ao analisar a pontuação obtida pelas crianças participantes da pesquisa na prova de Resolução de Conflito, nota-se que não houve diferença estatística significativa entre as idades; o que está de acordo com o descrito na literatura; este dado sugere que as crianças em idade escolar resolvem conflitos da mesma maneira ${ }^{(1)}$.

A maioria dos participantes utilizou estratégias do Nível 2, ou seja, sugeriram soluções unilaterais para resolução de conflitos, não levando em consideração o outro indivíduo, talvez crianças mais velhas e adolescentes utilizem com mais freqüência estratégias mais sofisticadas, como as soluções cooperativas e mútuas, sendo necessário então ampliar, em estudos futuros a faixa etária investigada.

Na prova de Resolução de Conflito, as meninas obtiveram uma pontuação média maior do que os meninos, o que não era esperado e difere dos dados relatados na literatura ${ }^{(1)}$. Provavelmente essa diferença tenha sido influenciada pelo maior número de meninas participantes, $65 \%$ da amostra total; da mesma forma, o estudo deverá ser replicado, igualando o número de participantes por sexo.

Na prova de Fluência a ocorrência de disfluências comuns variou de 4 a 24, sendo que a média de ocorrência de disfluências comuns nesta pesquisa foi 10. Os sujeitos S12 e S15 realizaram discursos fluentes, com apenas 4 disfluências comuns e obtiveram na prova de Resolução de Conflito 5 e 13 pontos respectivamente. O sujeito 2 realizou um discurso disfluente, com 24 disfluências comuns e obteve 9 pontos na prova de Resolução de Conflito. Estes dados mostram que, no caso dessas crianças, não houve relação entre o desempenho nas duas tarefas.

De fato, ao analisar as tarefas de todas as crianças, não se observou correlação estatística significante entre a pontuação na prova de Resolução de Conflito e a ocorrência de disfluências comuns.

Não há na literatura trabalhos que investigaram a relação entre disfluências comuns e resolução de conflito; porém, há diversos estudos que mostram uma importante relação entre linguagem e fluência ${ }^{(15-20)}$, que foi o que motivou nosso interesse na execução do presente estudo. Entretanto, nesta pesquisa, não foi possível estabelecer uma relação direta entre as duas variáveis.

Era esperado que ocorresse uma correlação entre a pontuação na prova de Resolução de Conflito e a ocorrência de disfluências comuns (discurso fluente), pois as duas habilidades

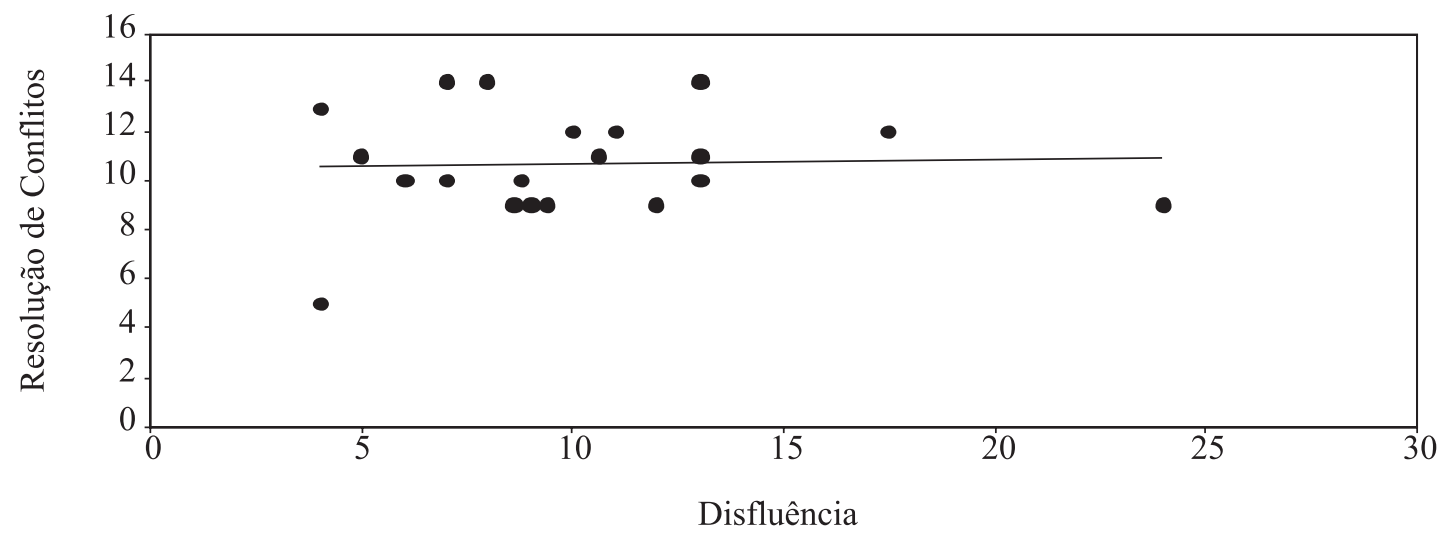

Figura 2. Pontuação total na prova de Resolução de Conflito e ocorrência de disfluências comuns 
compartilham de processos subjacentes, como: a capacidade de elaboração de uma resposta ou discurso e a capacidade de expressar suas idéias, seja na forma de uma resposta ou na forma de narrativa. Entretanto, no presente estudo, não foi possível estabelecer uma correlação direta entre as duas variáveis.

Alguns fatores podem ter contribuído para os resultados que encontramos; um deles pode ser o tipo de prova que utilizamos para avaliar a fluência, que difere em forma, da prova de Resolução de Conflito, ou seja, o discurso era eliciado a partir de uma figura, não havia um desencadeador de questões. Este aspecto não foi considerado no desenho inicial do estudo, necessitando também de investigação, ou ainda, que se refaça a análise da fluência a partir das respostas dadas na própria prova de Resolução de Conflito, eliminando assim alguma variável que o tipo de coleta possa desencadear.

Outro aspecto que podemos destacar refere-se ao fato que hipotetizamos que haveria uma relação entre habilidades de resolução de conflito e fluência a partir dos indicativos de literatura $^{(18,20-21)}$, mas nossos achados não confirmaram essa hipótese. Talvez sejam necessários outros estudos, para que possamos efetivamente refutar essa hipótese; pode ser que da forma como, até o momento, temos condições de medir estas duas habilidades, não seja possível a confirmação de relação entre elas.

Cabe ainda destacar que, embora haja indicativos de lite- ratura que habilidades de fala e linguagem se correlacionem para a efetividade discursiva ${ }^{(15-19)}$, os estudos são ainda muito iniciais e necessitamos de mais tempo e mais pesquisas para confirmar, ou não, se os processamentos de fala e linguagem, que na comunicação são diretamente relacionados, correlacionam-se quando medidos separadamente. Acreditamos que a realização de estudos comparando as performances (fala e linguagem) a partir da mesma prova, como sugerimos acima, possam indicar se há pertinência, ou não, na continuidade desse tipo de investigação.

\section{CONCLUSÕES}

Na prova de Resolução de Conflito, as crianças utilizaram principalmente estratégias pertencentes ao Nível 2, ou seja, soluções unilaterais para resolução de conflitos e as crianças não utilizaram estratégias mais sofisticadas com o passar da idade.

Ao comparar o desempenho das crianças na prova de Resolução de Conflito à ocorrência de disfluências comuns, não foi possível estabelecer uma relação direta entre as duas variáveis.

Sugere-se a realização de novos estudos para a confirmação, ou não, de nossos achados.

\begin{abstract}
Purpose: The aims of this study were: (1) to investigate conflict resolution (CR) abilities in normally developing school-aged children; (2) to verify whether there is a relationship between the occurrence of normal disfluencies (ND) and the development of conflict resolution abilities. Methods: Twenty normally developing children, whose ages ranged between seven and ten, participated in this study. The participants underwent a language screening test, covering the following aspects: phonology, phonological awareness abilities, reading and writing skills. Those who performed within the expected for their chronological ages were assessed through a CR test and a fluency test. The answers given in the CR test were grouped into 28 categories and then scored. In the fluency test the occurrence of normal disfluencies (ND) was verified. Results: The participants scored from 5 to 14 points $(M=10,7)$ in the CR test, nevertheless it was not observed any correlation between final scores in that test and chronological age $(\mathrm{p}=0,361)$. In the fluency test, the occurrence of ND varied from 4 to $24(\mathrm{M}=10)$. It was not noticed any statistically significant correlation between the performance in the CR test and the occurrence of ND ( $\mathrm{p}=0,899)$. Conclusions: In the CR test, in order to solve the conflict, children used mainly unilateral strategies. It was also observed that the strategies did not improve with age. Regarding the comparison between the performance in the CR test and the occurrence of ND, it was not possible to establish direct relationship between these variables.
\end{abstract}

Keywords: Cognition; Child language; Language development; Communication; Speech

\section{REFERÊNCIAS}

1. Stevens LJ, Bliss LS. Conflict resolution abilities of children with specific language impairment and children with normal language. J Speech Hear Res. 1995;38(3):599-611.

2. Locke JL. A theory of neurolinguistic development. Brain Lang. 1997;58(2):265-326.

3. Skuse DH, James RS, Bishop DV, Coppin B, Dalton P, Aamodt-Leeper $\mathrm{G}$, et al. Evidence from Turner's syndrome of an imprinted X-linked locus affecting cognitive function. Nature. 1997;387(6634):705-8. Comment in: Nature. 1997;387(6634):652-3.

4. Horowitz L, Jansson L, Ljungberg T, Hedenbro M. Behavioural patterns of conflict resolution strategies in preschool boys with language impairment in comparison with boys with typical language development.
Int J Lang Commun Disord. 2005;40(4):431-54.

5. Mostow AJ, Izard CE, Fine S, Trentacosta CJ. Modeling emotional, cognitive, and behavioral predictors of peer acceptance. Child Dev. 2002;73(6):1775-87.

6. Horowitz L, Jansson L, Ljungberg T, Hedenbro M. Interaction before conflict and conflict resolution in pre-school boys with language impairment. Int J Lang Commun Disord. 2006;41(4):441-66.

7. Brinton B, Spackman MP, Fujiki M, Ricks J. What should chris say? The ability of children with specific language impairment to recognize the need to dissemble emotions in social situations. J Speech Lang Hear Res. 2007;50(3):798-811.

8. Conti-Ramsden G, Botting N. Social difficulties and victimization 
in children with SLI at 11 years of age. J Speech Lang Hear Res. 2004;47(1):145-61

9. Hart KI, Fujiki M, Brinton B, Hart CH. The relationship between social behavior and severity of language impairment. J Speech Lang Hear Res. 2004;47(3):647-62.

10. Farmer M. Language and social cognition in children with specific language impairment. J Child Psychol Psychiatry. 2000;41(5):627-36.

11. Craig HK, Evans JL. Pragmatics and SLI: within-group variations in discourse behaviors. J Speech Hear Res. 1993;36(4):777-89.

12. Andrade CRF. Processamento da fala: aspectos da fluência. Pró-Fono. 2000;12(1):69-71.

13. Meira I. Abordagem psicolingüística da gagueira. In: Ferreira LP, BefiLopes DM, Limongi SCO, organizadores. Tratado de fonoaudiologia. São Paulo: Editora Rocca; 2004. p. 1017-26.

14. Andrade CRF. Fluência. In: Andrade CRF, Befi-Lopes DM, Fernandes FDM, Wertzner HF. ABFW: teste de linguagem infantil nas áreas de fonologia, vocabulário, fluência e pragmática. 2a ed. rev. ampl. e atual. Barueri: Pró-Fono; 2004.

15. Trautman LS, Healey EC, Norris JA. The effects of contextualization on fluency in three groups of children. J Speech Lang Hear Res. 2001;44(3):564-76.

16. Hall NE. Lexical development and retrieval in treating children who stutter.

Lang Speech Hear Serv Sch. 2004;35(1):57-69. Comment in: Lang Speech Hear Serv Sch. 2005;36(2):152-6; author reply 156-9.

17. Weiss AL. What child language research may contribute to the understanding and treatment of stuttering. Lang Speech Hear Serv Sch. 2004;35(1):30-3. Review.
18. Befi-Lopes DM. Avaliação, diagnóstico e aspectos terapêuticos nos Distúrbios Específicos de Linguagem. In: Ferreira LP, Befi-Lopes DM, Limongi SCO, organizadores. Tratado de fonoaudiologia. São Paulo: Editora Rocca; 2004. p. 987-1000.

19. Boscolo B, Ratner NB. Fluency of school-aged children with a history of specific expressive language impairment: an exploratory study. Am J Speech Lang Pathol. 2002;11(1):41-9.

20. Bliss LS, McCabe A, Miranda AE. Narrative Assessment Profile: discourse analysis for school-age children. J Commun Disord. 1998;31(4):347-62; quiz 363.

21. McCabe A, Rollins PR. Evaluating narrative discourse skills. In: Cole KN, Dale PS, Thal DJ, editors. Assessment of communication and language. Baltimore: P.H. Brookes; c1996.

22. Wertzner HF. Fonologia. In: Andrade CRF, Befi-Lopes DM, Fernandes FDM, Wertzner HF. ABFW: teste de linguagem infantil nas áreas de fonologia, vocabulário, fluência e pragmática. $2 \mathrm{a}$ ed. rev. ampl. e atual. Barueri: Pró-Fono; 2004.

23. Herrero SF. Perfil das crianças: pré-escolares e escolares no teste de sensibilidade fonológica. (Dissertação Mestrado). São Paulo: Faculdade de Filosofia, Letras e Ciências Humanas da Universidade de São Paulo; 2001.

24. Rosal CAR. Habilidades de segmentação fonêmica em crianças normais de primeira, segunda e terceira séries do ensino fundamental [dissertação]. São Paulo: Faculdade de Filosofia, Letras e Ciências Humanas da Universidade de São Paulo; 2002.

25. Andrade CRF, Befi-Lopes DM, Fernandes, FDM, Wertzner HF. Manual de avaliação de linguagem do serviço de Fonoaudiologia do centro de saúde escola Samuel B. Pessoa. São Paulo; 1997. 127f. [Publicação Interna].

Anexo 1. Situações hipotéticas de conflito

\footnotetext{
1 - Thiago é o melhor amigo de João. Mas agora Thiago brinca com um novo menino da escola todos os dias. Thiago nunca mais brinca com João.

2 - Rodrigo quer usar o computador para jogar seu jogo favorito. Seu irmão Lucas já está usando o computador. Lucas odeia ser interrompido quando está usando o computador.

3 - Marcelo está com fome. Ele quer que seu irmão mais velho, Daniel, o ajude a pegar alguns biscoitos que estão em uma prateleira alta. Marcelo está com medo que Daniel diga não.

4 - Tem um menino chamado Marcos que mora perto do Eduardo. Marcos é muito chato. Quase todos os dias Marcos xinga Eduardo no caminho para escola.

5 - Pedro é um novo menino no bairro. Um sábado Bruno chama Pedro para assistir desenho animado. Depois de dez minutos Pedro muda de canal sem perguntar.
} 